\title{
Paced P wave morphology templates to guide atrial tachycardia localization
}

\author{
Ahmed El-Damaty ${ }^{1}$, Dhaifallah Yahya ${ }^{1}$, Mahmoud Elmowafy ${ }^{1}$, Hussein rizk $^{1}$, and Amir \\ AbdelWahab $^{2}$ \\ ${ }^{1}$ Cairo University \\ ${ }^{2}$ Queen Elizabeth II Health Sciences Center
}

June 10, 2020

\begin{abstract}
Introduction: Surface ECG is a useful tool to guide mapping of focal atrial tachycardia (AT).We thought to construct an algorithm, based on paced $\mathrm{P}$ wave templates from different anatomical sites in both atria in patients with normal hearts. Methods: We prospectively enrolled consecutive patients who underwent electrophysiology study, having no heart disease. Atrial pacing was carried out at different anatomical sites in both atria. Paced P wave morphology (PWM) and duration (PWD) were assessed. P wave morphology was classified into: positive, negative, biphasic (+/- or -/+) and isoelectric. A proposed algorithm was generated from the constructed templates of each pacing site. Results: Sixty-four patients (25 males) were enrolled. Mean age was $37 \pm 13$ years. Atrial pacing was performed in 61 patients (95\%) at the right atrium and in 15 patients $(23 \%)$ at the left atrium. A neg/iso P wave in V1, a pos/iso P wave in AVL and lead I identified right atrial pacing sites $(\mathrm{p}=0.01, \mathrm{p}=0.02$ and $\mathrm{p}=0.02$, respectively). Negative $\mathrm{P}$ wave in lead aVL identified left pulmonary veins when compared to right pulmonary veins $(\mathrm{P}=0.03)$. $\mathrm{PWD}$ was significantly longer when pacing from lateral tricuspid annulus (TA) as compared to medial TA $(136 \pm 12 \mathrm{~ms}$ vs. $99 \pm 10 \mathrm{~ms}, \mathrm{P}=<0.001)$ and when pacing from the left superior pulmonary vein as compared to the right superior pulmonary vein $(152 \pm 12 \mathrm{~ms}$ vs. $135 \pm 10 \mathrm{~ms},(\mathrm{P}=0.001)$. Conclusions: PWM and PWD derived from templates generated through atrial pacemapping could be used to guide localization of focal AT.
\end{abstract}

\section{Hosted file}

Manuscript submission.pdf available at https://authorea.com/users/332132/articles/458564paced-p-wave-morphology-templates-to-guide-atrial-tachycardia-localization 